\title{
INSECT CLASSIFICATION USING SQUEEZE-AND-EXCITATION AND ATTENTION MODULES - A BENCHMARK STUDY
}

\author{
Yoon Jin Park, Gervase Tuxworth, Jun Zhou
}

School of Information and Communication Technology, Griffith University, Australia

\begin{abstract}
Insect recognition at the species level is an active research field with a variety of applications. With the advancement of convolutional neural networks an automatic fine-grained image classifier has displayed encouraging performance. Despite these recent advances, differentiating images at the species level is still a challenge. To address the problems arising from insect-specific peculiarities, this paper presents a novel network that consists of squeeze-and-excitation modules and attention modules, enabling the network to focus on more informative and differentiating features with a limited number of training iterations and a small dataset. The proposed model is trained on an insect dataset collected from Atlas of Living Australia. The results reveal that the integrated model achieves higher accuracy than several alternative methods on the introduced insect dataset.
\end{abstract}

Index Terms - Insect image classification, fine-grained image recognition, gating and attention enhanced networks

\section{INTRODUCTION}

Insect classification has been used in many beneficial projects aiming to achieve sustainability and promote bio-diversity. One of the important recent efforts in this category involves documenting existing or emerging living species and their associated properties, such as a taxonomy, an occurrence observation or a habitat, or any changes across a different time line and location. The large collection of these types of data including images is notable through examples of citizen science projects over the world, such as iNaturalist ${ }^{1}$, and BugGuide $^{2}$. Despite these efforts, a curated dataset for an insect image recognition task at the species level is scarce and most images in the existing datasets tend to be categorized with the mixed sub-class of order, family, genus, and species. To enable the collection and analysis of a large set of data, insect image recognition as the first step forward could help citizen scientists to capture and classify insect images at the species level with relevant records.

In particular, the classification task at the species level is particularly relevant in collecting and storing data, tracking

\footnotetext{
${ }^{1}$ https://www.inaturalist.org/

${ }^{2}$ https://bugguide.net/node/view/15740
}

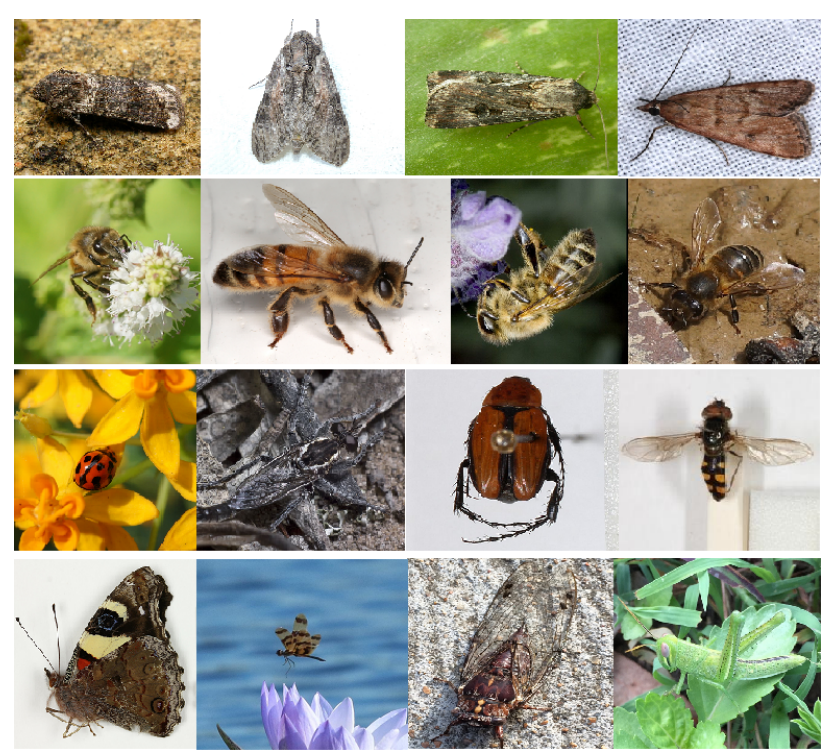

Fig. 1: The complexities of the insect classification problem. First row: Similarity between species from the collected insect dataset (Agrotis porphyricollis, Agrius convolvuli, Agrotis munda, Achyra affinitalis). Second row: Same specie with different appearances (Apis Mellifera). Third and fourth Row: Challenging images due to orientation, pose and background.

trends and monitoring signs of significant drops in insect species to evidence the subsequent changes in biomass [1]. Previous studies adopt automatic image recognition approaches for a variety of purposes: insect classification using a traditional convolutional neural network [2], detection and classification of crop insects [3], segmentation and counting in the Insect Soup Challenge [4], image tracking of moving insects [5], and beetle identification at the species level for potential food contamination [6]. The iNat2017 and iNat2018 competitions [7] aim to push the benchmark of an automatic image classification of living species in a realworld setting. Recent advances in image classification using convolutional neural networks (CNNs) [8], such as Inception [9], ResNet [10], and ResNext [11], facilitating image recognition tasks for a broad range of purposes requiring the processing and analysis of a large volume of data.

In this paper, we focus on collecting indoor and outdoor photos of insect species from Atlas of Living Australia 


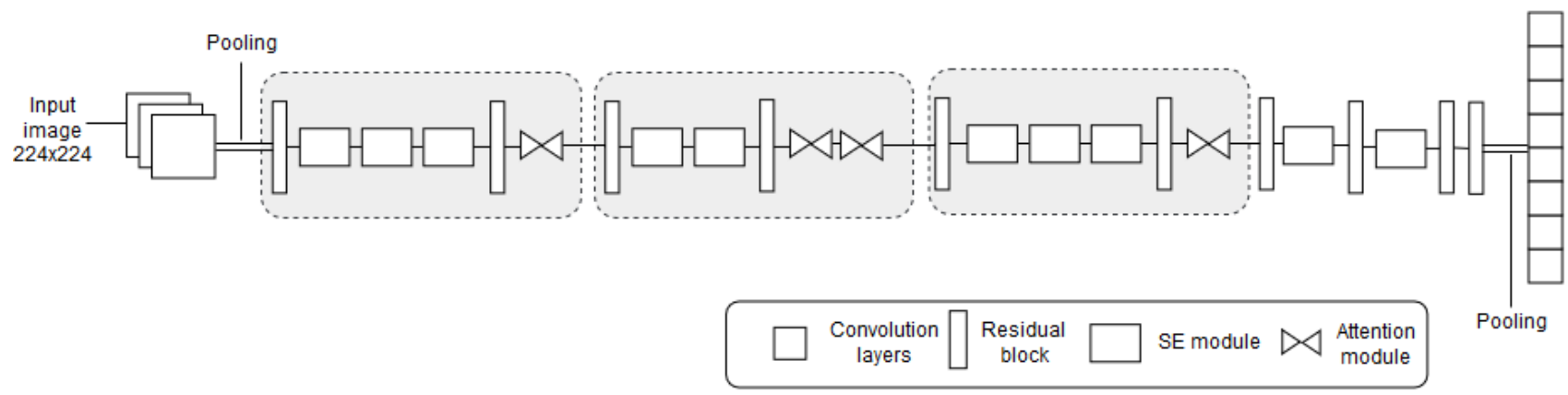

Fig. 2: Example architecture of the proposed model

$(\mathrm{ALA})^{3}$ and presenting a fine-grained insect image recognition system as a benchmark. This dataset contains 34,525 images for 123 classes of species. They cover a variety of scenarios including insects in different sizes, illumination conditions, and background, imposing a high challenge in the recognition task.

As seen in Fig. 1, classification at the species level requires very fine and detailed information for visually-similar species. Certain insect species appear to be alike to nonexperts. Insect images from a single species can also appear to be different based on their pose and deformation, indicating the orientation or posture that images are taken which may cause a large variation for a particular insect.

These characteristics motivate us to focus on an insect classifier that can capture useful and localized features in order to differentiate classes at the species level. To address these challenging problems, we propose to develop a novel network that integrates squeeze-and-excitation (SE) modules and attention modules into a ResNet-based classifier [10]. This aims to make efficient use of limited computational resources to obtain more informative feature maps within short iterations of training.

\section{INSECT DATASET}

Collecting a moderate-to-large dataset for insect images at the species level is challenging because existing insect datasets are generally unbalanced [7], labelling and validating images for a new dataset requires special skills acquired from extensive experience, and the number of species in insects is countless while many undiscovered species emerge over time [12].

The insect dataset at the species level is collected from ALA website which aims to establish and maintain biodiversity data from multiple sources including citizen science projects. Much of the information in the ALA consists of Australian species, ranging from fauna and plants to microorganisms. Records consist of an overview of resources, gallery, names (accepted/common name), classification, occurrences, literature, and sequences. In particular, classification fields

\footnotetext{
${ }^{3}$ https://www.ala.org.au/
}

are categorized from kingdom through family and genus to species level, which is our focus for the data collection.

Through the ALA API, we collected the list of insects with the species filter. The number of species under Insects that have image urls makes up 16,396 as of October 2018. The species are sorted based on the number of occurrences where the species have images, and images for the top 200 species with this criterion are downloaded.

After downloading total 34,789 images, we removed images not including a specific insect, providing the final number of 34,525 images. The labels for insect images are selected from the 'species' field from the records. During the review of each image, indoor insect images were cropped so as not to include labels of information regarding the photographed insect (for example, information about species name or the location it was found). This resulted in 123 species in the insect dataset. The number of images in the dataset displays a large disparity between species, where the top species contains over $4 \%$ of the training images.

\section{METHODOLOGY}

\subsection{Model}

Typically, to enhance the accuracy of image classification tasks, CNNs are used in conjunction with numerous epochs of training on a relatively large dataset. To address the challenges of an insect classification task, we develop an image classifier that can focus on more useful features during a limited number of training epochs with a smaller dataset. The proposed model uses a ResNet as the backbone. SE modules and attention modules are integrated into the structure for fine-grained feature extraction, as shown in Fig. 2.

The first block of the model uses three layers of a small receptive field of a $3 \times 3$ kernel, like VGGNets [13], to obtain the effect of a $7 \times 7$ kernel while reducing the number of parameters. A max pooling layer is then used followed by the residual block. The residual block consists of three convolutional layers, each of which feeds-forward with Batch Normalization [14] and rectification units(ReLU) [15]. The residual blocks [10] are alternated with other modules to collect 
spatial informative features reliably through the model.

To enhance discriminative feature representation in an efficient manner, the proposed network integrates the gating and attention mechanisms found in squeeze-and-excitation (SE) [16] and attention modules [17]. SE modules and attention modules are alternated in blocks, with the residual blocks used in-between to reduce instability arising from splitting and combining two different modules. The last two layers of SE modules are added to capture more class-relevant informative features in the model.

\subsection{SE Modules}

Gating and top-down attention mechanisms in a classifier have been used for passing top information for feature learning to the next stage with Region Proposal Networks [18], updating control gates with top information that can influence the feed-forward process [19], or processing sequential learning to determine how to retrain previous informative features through gate mechanisms [20].

In the proposed method, the SE module contains a component where global spatial information gets squeezed into channel embedding, and then these global statistics are feedforwarded through excitation, comprised of two convolutional layers with a ReLU in between, and finally passed though a Sigmoid (gating) activation function. We specifically adopt the SE-ResNet module to incorporate the properties of identity mapping [21] in the proposed model.

SE modules contribute to the proposed model by collecting an insect image's contextual information for each channel and the channel-wise dependencies are then explored to control what information is to be emphasized. This mechanism specifically helps to increase differentiating capacity of the proposed model to alleviate the problems associated with inter-class similarity of insects.

\subsection{Attention Modules}

Attention mechanisms can also help to develop a localized understanding of images [22, 23]. Recent developments [24, 25] utilizing bottom-up and top-down attention mechanisms with the lateral connections in segmentation tasks have been adopted in image classification problems [17], showing potential improvements in accuracy in a more timely fashion. We therefore propose to improve insect classification by integrating attention into a ResNet-based classifier.

The attention module integrates the soft attention mechanism (a bottom-up and top-down mask branch) with residual blocks (a trunk branch) within an individual feed-forward process. The down-sampling layers summarize global information while the up-sampling distributes global information to an appropriate position in the output layers where useful features are also mapped with lateral connections.

Attention module enables the proposed model to capture localized features of a specific insect species by incorporating soft attention masks. Through this mechanism, semantic information with dense features are passed together through the network, aiming to focus on fine-grained and useful areas in an insect image.

\section{EXPERIMENTS AND ANALYSIS}

In this section, we describe the experiments undertaken to show the performance of the proposed integrated classifier on the collected insect dataset. In addition, we investigate how the components of the proposed integrated classifier influence the performance differently through additional studies.

\subsection{Setting}

In our experiments, the images were resized to 224 x 224 with random cropping or padding and augmented with a horizontal or vertical flip, lighting, and zoom variations. We used a standard practice to normalize RGB channels by subtracting mean values and dividing them by standard deviation values. We randomly split the dataset using $70 \% / 15 \% / 15 \%$ ratios for each insect species for training, validation, and testing sets, resulting in 24,287 training samples, 5,119 validation samples, and 5,119 testing samples respectively.

For the proposed method, we generated two versions: SERAN and SERAN-D. SERAN is the model with the smaller number of parameters while SERAN-D is a deeper network. We compare our method against widely used networks. To implement and train our network, we utilize general best practices found in previous studies, including batch normalization [14], dropout [8], and one of the Adam optimization methods [26]. All networks are trained with AdamW [27] with weight decay set to 0.01 . The initial learning rate used on the collected insect dataset was 5e-04. The models were implemented with Pytorch and version 1 of the fast.ai library. The models were trained on one Titan P100 GPU with Google Cloud Platform's standard machine type with 8 vCPUs and 30 GB of memory.

\subsection{Results \& Discussion}

Table 1 compares the validation and testing errors on the collected insect dataset by different models. Without the pretrained weights, ResNet-101 and ResNet-152 display lower performance as these networks appear to require a greater number of epochs where there is a limited number of the training samples. This implies the effectiveness of SE and attention modules that help to focus on useful information during a small number of training iterations.

SERAN and SERAN-D perform better than Attention-92 (Residual Attention Network, i.e. RAN) and SE-Resnet-101 networks (both networks re-implemented with Pytorch), with SERAN-D showing a $5.31 \%$ and $3.79 \%$ reduction on top-1 


\begin{tabular}{c|c|c|c|c}
\hline & \multicolumn{2}{|c|}{ Validation } & \multicolumn{2}{c}{ Testing } \\
\hline Model & Top-1 err. (\%) & Top-5 err. (\%) & Top-1 err. (\%) & Top-5 err. (\%) \\
\hline \hline ResNet-101 [10] & 25.18 & 8.63 & 24.64 & 8.48 \\
\hline ResNet-152 [10] & 28.36 & 9.98 & 28.70 & 10.49 \\
\hline Attention-92 (RAN) [17] & 17.25 & 5.14 & 18.89 & 5.74 \\
\hline SE-Resnet-101 [16] & 18.52 & 5.72 & 17.37 & 6.23 \\
\hline SERAN & 15.78 & 4.77 & 15.28 & 4.53 \\
\hline SERAN-D & $\mathbf{1 3 . 2 4}$ & $\mathbf{3 . 8 2}$ & $\mathbf{1 3 . 5 8}$ & $\mathbf{4 . 0 4}$ \\
\hline
\end{tabular}

Table 1: Top-1 and Top-5 errors on the validation and testing datasets.

\begin{tabular}{c|c|c}
\hline Model & Top-1 err. (\%) & Top-5 err. (\%) \\
\hline \hline SERAN-D with Max pooling & $\mathbf{1 3 . 2 4}$ & $\mathbf{3 . 8 2}$ \\
\hline SERAN-D with AVG pooling & 15.84 & 4.65 \\
\hline SERAN-D RAN only, removing all SE modules & 19.65 & 5.72 \\
\hline SERAN-D SE only, removing all RAN modules & 16.06 & 4.74 \\
\hline SERAN, removing one RAN and six SE modules from SERAN-D & 15.78 & 4.77 \\
\hline
\end{tabular}

Table 2: Top-1 and Top-5 error of the modified models on the validation dataset

errors and a $1.70 \%$ and $2.19 \%$ reduction on top-5 errors on the testing dataset, respectively.

We further compared SERAN-D with SENet-154 that won first place in ILSVRC 2017 classification competition [16]. Despite the smaller size of SERAN-D where the number of the parameters is $2.16 \times 10^{7}$ less than SENet-154, the accuracy of the SERAN-D model is comparable with the SENet-154 which achieved $13.33 \%$ and $3.99 \%$ on top-1 and top-5 errors respectively.

Table 2 shows the ablation study of the effect of different layers and modules in the SERAN-D network. First, we investigate the effect of different pooling layers in the proposed network by replacing max pooling layers with average pooling layers. Max pooling layers in the overall model and attention Modules perform better, leading to the selection of max pooling layers within the proposed model.

We experiment the role of channel-wise global information generated and shared by SE modules in the SERAN-D model, by replacing the SE blocks with the residual blocks. Without the SE blocks, the accuracy of the modified network declines by $3.59 \%$ (top-1) and $0.98 \%$ (top-5) compared to the model with Attention module only, indicating the importance of re-calibrating and passing the informative features originating from the SE modules.

Attention modules appear to play an important role in training reliably. Without attention modules, the modified model starts to show signs of over-fitting after 20 epochs as opposed to a lack of such symptom in the modified model with Attention modules only.

Further analysis reveals that removing one attention module and six SE modules from the SERAN-D model can reduce the number of parameters significantly (a reduction of $2.87 \times 10^{7}$ ), without resulting in a huge loss of performance.
This simplified model is equivalent to SERAN in Table 1.

From the displayed outputs, it is observed that the integration of attention and SE modules leads to better performance in insect classification at the species level with the significantly less number of parameters. As the SE modules help to produce better accuracy while attention modules help to increase performance reliably, the SERAN-D network integrated ten SE blocks with four attention modules.

\section{CONCLUSION}

In this paper, the challenges in the insect classification task were investigated through an integrated classifier on the insect dataset collected from ALA. The proposed SERAN-D classifier incorporates squeeze-and-excitation blocks and attention modules to address the problems arising from insect-specific characteristics such as inter-species similarity and a variation within a certain insect species due to pose and deformation. Future research will investigate how to efficiently recognize species by addressing the problems associated with a large variation due to pose and deformation.

\section{REFERENCES}

[1] B. C. Lister and A. Garcia, "Climate-driven declines in arthropod abundance restructure a rainforest food web," PNAS, vol. 115, no. 44, pp. E10397-E10406, 2018.

[2] S. Lim, S Kim, and D. Kim, "Performance effect analysis for insect classification using convolutional neural network," in IEEE ICCSCE, 2017.

[3] D. Xia, P. Chen, B. Wang, J. Zhang, and C. Xie, "Insect detection and classification based on an improved 
convolutional neural network," Sensors, vol. 18(12), pp. 4169, 2018.

[4] K. Mele, "Insect soup challenge: Segmentation, counting, and simple classification," in ICCV Workshop, 2013, pp. 168-171.

[5] B. Risse, M. Mangan, L. Del Pero, and B. Webb, "Visual tracking of small animals in cluttered natural environments using a freely moving camera," in ICCV Workshop, 2017, pp. 2840-2849.

[6] D. Martin, H. Ding, L. Wu, H. Semey, A. Barnes, D. Langley, SI. Park, Z. Liu, W. Tong, and J. Xu, "An image analysis environment for species identification of food contaminating beetles," in AAAI, 2016, pp. 43754376.

[7] G. Van Horn, O. Mac Aodha, Y. Song, Y. Cui, C. Sun, A. Shepard, H. Adam, P. Perona, and S Belongie, "The inaturalist species classification and detection dataset," in $C V P R, 2018$, pp. 8769-8778.

[8] A. Krizhevsky, I. Sutskever, and G. E. Hinton, "Imagenet classification with deep convolutional neural networks," in NIPS, 2012, pp. 1097-1105.

[9] C. Szegedy, W. Liu, Y. Jia, P. Sermanet, S. E. Reed, D. Anguelov, D. Erhan, V. Vanhoucke, and A. Rabinovich, "Going deeper with convolutions," in CVPR, 2015, pp. 1-9.

[10] K. He, X. Zhang, S. Ren, and J. Sun, "Deep residual learning for image recognition," in CVPR, 2016, pp. 770-778.

[11] S. Xie, , R. Girshick, Z. Dollar, P. Tu, and K. He, "Aggregated residual transformations for deep neural networks," in CVPR, 2017, pp. 5987-5995.

[12] M. Martineau, D. Conte, R. Raveaux, I. Arnault, D. Munier, and G Venturini, "A survey on image-based insect classification,” $P R$, vol. 65, pp. 273-284, 2017.

[13] K. Simonyan and A. Zisserman, "Very deep convolutional networks for large-scale image recognition," in ICLR, 2015.

[14] S. Ioffe and C. Szegedy, "Batch normalization: Accelerating deep network training by reducing internal covariate shift," in ICML, 2015, pp. 448-456.

[15] V. Nair and G. E. Hinton, "Rectified linear units improve restricted boltzmann machines," in ICML, 2010, pp. 807-814.

[16] J. Hu, L. Shen, and G. Sun, "Squeeze-and-excitation networks," in CVPR, 2018, pp. 7132-7141.
[17] F. Wang, M. Jiang, C. Qian, S. Yang, C. Li, H. Zhang, $\mathrm{X}$. Wang, and X. Tang, "Residual attention network for image classification,” in CVPR, 2017, pp. 3156-3164.

[18] S. Ren, K. He, R. Girshick, and J Sun, "Faster r-cnn: towards real-time object detection with region proposal networks," in NIPS, 2015, pp. 91-99.

[19] C. Cao, X. Liu, Y. Yang, Y. Yu, J. Wang, Z. Wang, Y. Huang, L. Wang, C. Huang, W. Xu, and T.S. Ramanan, D.and Huang, "Look and think twice: Capturing top-down visual attention with feedback convolutional neural networks," in ICCV, 2015, pp. 2956-2964.

[20] V. Mnih, N. Heess, A. Graves, and K. Kavukcuoglu, "Recurrent models of visual attention," in NIPS, 2014, pp. 2204-2212.

[21] K. He, X. Zhang, S. Ren, and J. Sun, "Identity mappings in deep residual networks," in ECCV, 2016, pp. 630645 .

[22] M. Jaderberg, K. Simonyan, A. Zisserman, and K. Kavukcuoglu, "Spatial transformer networks," in NIPS, 2015, pp. 2017-2025.

[23] K. Li, Z. Wu, K-C. Peng, J. Ernst, and Y. Yun Fu, "Tell me where to look: Guided attention inference network," in CVPR, 2018, pp. 9215-9223.

[24] O. Ronneberger, P. Fischer, and T. Brox, "U-net: Convolutional Networks for Biomedical Image Segmentation," in MICCAI, 2015, pp. 234-241.

[25] TY. Lin, P. Dollr, R. Girshick, K. He, B. Hariharan, and S. J. Belongie, "Feature pyramid networks for object detection," in CVPR, 2017, pp. 936-944.

[26] D. P. Kingma and J. Ba, "Adam: A method for stochastic optimization," in ICLR, 2014.

[27] I. Loshchilov and F. Hutter, "Decoupled weight decay regularization," in ICLR, 2019. 